\title{
Threshold Effects of Creative Problem-Solving Attributes on Creativity in the Math Abilities of Taiwanese Upper Elementary Students
}

\author{
Chia-Yi Lin \\ Office of Research and Development, National Cheng Kung University, No. 1 University Road, Tainan City, Taiwan \\ Correspondence should be addressed to Chia-Yi Lin; joyvhsui@gmail.com
}

Received 23 March 2017; Revised 4 July 2017; Accepted 2 August 2017; Published 19 September 2017

Academic Editor: Kirsi Tirri

Copyright @ 2017 Chia-Yi Lin. This is an open access article distributed under the Creative Commons Attribution License, which permits unrestricted use, distribution, and reproduction in any medium, provided the original work is properly cited.

\begin{abstract}
This study aimed to help determine what the typology of math creative problem-solving is. Different from studies that have discussed the threshold effect between creativity and intelligence, this research investigated the threshold effect between creativity and other attributes. The typology of the math creative problem-solving abilities of 409 fifth-and sixth-grade Taiwanese students was identified and compared in this study. A Creative Problem-Solving Attribute Instrument was devised for this study, with the aim of measuring students' perceptions on their motivation, knowledge, and skills, both in general and in specific domains. Divergent and convergent thinking were also measured. Cluster analyses yielded three creative problem-solving typologies: High, Medium, and Low. The High Attribute group scored significantly higher in the Math Creative Problem-Solving Ability Test than did the Medium Attribute and Low Attribute groups. The results suggest a threshold effect from several attributes-divergent thinking, convergent thinking, motivation, general knowledge and skills, domain-specific knowledge and skills, and environment-on students' creative problem-solving abilities. Balanced development of attributes may be an important consideration in nurturing creativity in children.
\end{abstract}

\section{Introduction}

Creative problem-solving is crucial for the improvement of society and is also important for the activities we do in our daily lives [1,2]. Additionally, creativity and the ability to solve problems creatively are considered essential skills that future talent must be equipped with [3]. In this study, creativity is a necessary component of creative problem-solving ability. It is therefore necessary to characterize the relationship between problem-solving (in general) and creativity. Creativity can be part of problem-solving [4-7]; it is especially so when solutions to problems are not easily reached or readily available and when the ideas required for solutions are novel and are particularly appropriate to solving the problems $[8$, 9]. Some researchers believe the process of creativity actually constitutes the whole of the problem-solving process, from problem finding to executing a plan [10]. Guilford [11] associated creativity with problem-solving and identified four stages of the creative process: (1) recognition of an existing problem; (2) production of a number of relevant ideas; (3) recognition of various possibilities produced; and (4) the drawing of appropriate conclusions that lead to the solution. Houtz [12] believed that Torrance's definition of creativity, defined as "the process of sensing gaps or disturbing, missing elements; forming ideas or hypotheses concerning them; testing these hypotheses and communicating the results, possibly modifying and retesting the hypotheses" $[13, p .16]$, is similar to the process of problem finding and problemsolving, because creativity can be exercised while solving open-ended and ill-defined problems.

It is believed that creative problem-solving ability is difficult to research and that it is difficult to put the results of that research into practice [14]. Various measures of creativity tend to have different operational definitions, and the attributes of creativity continue to be debated. During the early development of creativity research, the structure of creativity was measured via divergent thinking tests and has gradually come to be equated with divergent thinking [11, 13]. However, divergent thinking tests have been evidenced to have poor reliability and weak predictive validity [15]. 
Additionally, in some studies, the structure of creativity has been understood as an entity independent from academic achievement, which implies that knowledge and skills learned in school are not necessary in exercising creativity [1518]. However, with the emergence of the theory of domain specificity of creativity, the importance of knowledge and skills within specific domains has come to be recognized among researchers on creativity [19-21].

The foundation of this research is based on the belief that creativity is multifaceted and complex, and this belief has been evidenced in recent research [20, 22-29]. Guilford [22] claimed that several factors are involved in creative thinking: (a) sensitivity to the problem; (b) fluency; (c) novelty; (d) flexibility; (e) ability to synthesize; (f) analytical ability; (g) reorganization or redefinition; (h) complexity; and (i) evaluation. Torrance [28] discussed the multifaceted nature of creativity, defining creativity as "the process of sensing difficulties, problems, gaps in information, missing elements, something askew; making guesses and formulating hypotheses about these deficiencies; evaluating and testing these guesses and hypotheses; possibly revising and retesting them; and finally communicating the results" (p. 47). Amabile $[20,23]$ suggested a Componential Model of Creativity, which includes domain-relevant skills, creativityrelevant skills, and task motivation. Csikszentmihalyi [17] proposed six personal resources for producing creativity: intelligence, knowledge, styles of thinking, personality, motivation, and environmental context. Urban's [29] component model of creativity views the creative process as containing six components: (1) divergent thinking and acting; (2) general knowledge and base for thinking; (3) specific knowledge base and specific skills; (4) focus and task commitment; (5) motivation and motives; and (6) openness to and tolerance of ambiguity.

Cho $[24,30]$ proposed six attributes of creative problemsolving ability: divergent thinking; convergent thinking; motivation; general knowledge and skills; domain-specific knowledge and skills; and environment, in her Dynamic System Model of Creative Problem-Solving Ability, which was based upon Csikszentmihalyi's [17] complex model of creativity and Urban's [29] Componential Model of Creativity. Knowledge and skills provide individuals with preliminary resources in which divergent and convergent thinking either evaluate or search for alternatives [31,32]. Divergent thinking is used to search for alternatives. Convergent thinking is used to evaluate various alternatives against criteria and to correct errors in order to achieve the best answer to the problem $[21,33,34]$.

Cho suggested that creative problem-solving ability functions like an organic system whose attributes dynamically interact to solve a problem, where motivation, general knowledge and skills, and domain-specific knowledge and skills form the bases of creative problem-solving. Convergent and divergent thinking function as tools that utilize domainspecific and general knowledge and skills in order to solve problems in a new and useful fashion. Creative problemsolving ability can grow or diminish, depending on the micro- and macro-environmental conditions of the external organic systems individuals find themselves in. In addition, if any of the attributes are not developed to the necessary level, creativity will not blossom. Cho's model is the theory upon which this study is founded.

The threshold effect is one of the essential topics discussed in creativity research, and the correlation between creativity and intelligence is also often investigated. Creativity and creative problem-solving have also been stated to be related to intelligence [21]. The theory of the threshold effect for intelligence and creativity was first proposed by Guilford, who stated that creativity is well-predicted at IQ levels below 120 but not above 120 [21]. However, even when creativity is recognized as multifaceted and cannot be simply determined by a single factor, the threshold effect theory has merely been studied as it relates to intelligence and creativity but not applied to the correlations between creativity and other attributes. Torrance [13] claimed threshold effects in intelligence, but such effects have not yet been verified for other attributes of creativity.

In this study, the Creative Problem-Solving Attributes Inventory (CPSAI) was developed by combining Cho's dynamic system model [23, 24, 30] and Treffinger's CPS Model Version $4.0[35,36]$ as frames of reference. Version 4.0 of Treffinger's CPS emphasizes a three-stage process of solving problems creatively, where divergent thinking and convergent thinking are both utilized at each stage $[35,36]$. The three overall stages of the CPS process are as follows: understanding the problem (mess-finding, data-finding, and problem finding); generating ideas (idea-finding); and planning for action (solution-finding and acceptance-finding) [37]. Although it is not the latest version, CPS Version 4.0 clearly describes the creative problem-solving processes that young students, who are relatively inexperienced real-life problem solvers, might experience. It also does so in a less complex manner than is detailed in CPS Version 6.1, the latest one.

The main purpose of this study was to shed light on the essential attribute(s) of creative problem-solving ability in math, as based on Cho's theory. Most importantly, the study aimed to investigate whether there is a typology, or even a threshold effect, between creative problem-solving and a variety of attributes, among different levels of creative math problem-solving ability. This study aimed to provide insight into how best to nurture creativity.

\section{Materials and Methods}

2.1. Sample. A total of 409 fifth and sixth graders were recruited from two elementary schools in Taiwan. Because there is a minimum age requirement for Taiwanese students to enroll in elementary school and there is no accelerated program at the elementary level, fifth- and sixth-grade students' ages generally range from 10 to 12 years. 325 students $(54.8 \%$ males and $45.2 \%$ females) were sampled from School A. In School B, a sample of 84 students $(60.7 \%$ males and $39.3 \%$ females) participated. Demographically, these participants came from two-parent households (96\%), were born in Taiwan (99\%), and mainly spoke either Mandarin (59\%) or Taiwanese (37\%) at home. 
2.2. Research Questions. The following research questions were addressed in this study:

(i) How are the proposed attributes in Cho's theory related to corresponding attributes assessed by other established measures (e.g., the Creativity Assessment Packet, Critical Thinking Test, and Inventory of Parental Influence)?

(ii) What are the distinctive patterns of attribute combinations for creative problem-solving ability evidenced among fifth- and sixth-grade Taiwanese students?

(iii) After categorizing the respondents into three different groups based on their responses to the CPSAI, what were the differences between these three groups, in terms of their math creative problem-solving performance?

2.3. Instruments. The CPSAI is an instrument that measures students' self-perceptions of five attributes of creative problem-solving ability. To examine its structural validity, four other instruments were also administered: the Math Creative Problem-Solving Ability Test [26]; the Creativity Assessment Packet [38]; the Critical Thinking Test-I [39]; and the Inventory of Parental Influence [40]. The validity and reliability of each instrument are described below.

The CPSAI. The CPSAI is a self-report questionnaire in which students rate their frequency of using attributes of creative problem-solving, by responding to 41 items on a scale from 1 (Hardly Ever) to 5 (Very Often). The theoretical basis of the CPSAI is adopted from Cho's Dynamic System Model of Creative Problem-Solving and Treffinger's Creative ProblemSolving [23, 35]. There are five subscales included: Divergent Thinking; Convergent Thinking; Motivation; General Knowledge; and Environment. In each of the five subscales, the test questions describe possible behaviors for creative problem-solving attributes that can be exhibited during each stage of creative problem-solving. For instance, items in the Divergent Thinking subscale describe divergent thinkingrelated behaviors in the stages of problem finding (e.g., "I ask many related questions when I try to understand problems"), generating ideas (e.g., "I get many different ideas by thinking from different standpoints"), and planning for action (e.g., "I have several different procedures to solve problems").

Because the CPSAI is a new measure and the Math Creative Problem-Solving Ability Test (MCPSAT) is a slightly modified version of the original, these instruments were first piloted over a one-month period to establish reliability. Detailed information about the MCPSAT can be found in the next paragraph. The pilot group consisted of 74 students (31 fifth graders and 43 sixth graders) from various backgrounds, recruited from several private institutions across Tainan City and Tainan County. Cronbach's alpha for the test items showed reasonable internal-consistency on the subscales of Motivation ( $\alpha=.80)$, Environment $(\alpha=.88)$, Divergent Thinking $(\alpha=.89)$, and Convergent Thinking $(\alpha=.94)$ but only minimally adequate reliability on the subscale of General Knowledge and Skills $(\alpha=.65)$.
TABLE 1: Internal validity of the MCPSAT by item relevance indexes and item difficulty level.

\begin{tabular}{lccccccccc}
\hline Item & 1 & 2 & 3 & 4 & 5 & 6 & 7 & 8 & Mean \\
\hline $\begin{array}{l}\text { Infit } \\
\text { MnSq }\end{array}$ & .91 & 1.19 & 1.15 & .87 & 1.12 & .90 & 1.65 & 2.06 & 1.23 \\
Outfit & .87 & 1.05 & 1.01 & .86 & .93 & 1.13 & .99 & 2.06 & 1.11 \\
MnSq & & & & & & & & & \\
\hline Difficulty & -.63 & -.49 & -.37 & -.42 & -.11 & .53 & .74 & .76 & .00 \\
\hline
\end{tabular}

The Math Creative Problem-Solving Ability Test (MCPSAT). The MCPSAT is a creative problem-solving ability assessment of open-ended math problems that identifies gifted students in math [25]. Students' responses were evaluated in terms of fluency, flexibility, and originality [26]. Fluency is scored according to the number of correct answers to the problems. Flexibility is scored by the number of categories of correct answers produced for the problems. For instance, one of the questions is, "Using the following equation $(77 \times 15=$ $1155)$, please create up to 10 new equations." If a student's answer includes $15 \times 77=1155$ and $\sum_{i=1}^{15} 77$, this would be considered as two different categories. Originality is scored by how frequently a student's given correct answer to a question was provided by the other students (i.e., more frequent answers equal lower originality). A rubric with exemplary answers for each score ranking was developed by selecting and classifying all relevant responses to each question. The members of the research team discussed and revised the evaluation rubrics when faced with unique and challenging responses. Participants' responses were scored by math teachers who have been trained on the nature of the problems and on possible answers for each test item. Taiwanese math teachers reviewed the problems in terms of their difficulty, with comparative reference to the Taiwanese math curriculum, and noted students' difficulties during the pilot testing, resulting in the selection of eight problems (five easy items, two medium items, and one difficult item) for the final version of the MCPSAT for the study.

Statistical analyses were performed to examine the following aspects of the MCPSAT with regard to the current participants: internal-consistency reliability; internal validity; and difficulty level. Reliability, measured by internalconsistency, of the MCPSAT was found to be reasonably good, since Cronbach's alpha for the eight test items was .73. The internal validity of each test item was calculated using BIGSTEPS, and an item analysis was conducted based on Rasch's 1-parameter item-response model. The analysis model used in this study was the Partial Credit Model. The value for item 8 exceeded 2.0, which indicated low internal validity (see Table 1). Aside from item 8, all other internal validity indexes were less than 1.2. The overall fit statistics confirmed that the internal values of the items were high enough to be good. In this study, the item difficulty was calculated based on Rasch's 1-parameter item-response model. An item difficulty of 0.0 indicated average item difficulty. The higher the value, the more difficult the item. Results showed that the logit scores of items 7 and 8 were the most difficult items 
(see Table 1) and that the test items had been distributed from easy to difficult. Additionally, when all the items are distributed equally from easy to difficult, the ordering of the problems itself may motivate the participants to attempt and complete all of the problems in the instrument, rather than placing more difficult obstacles in their paths earlier on.

The Creativity Assessment Packet (CAP). The original version of the CAP was created by Williams [38]. Its Chinese version was translated and validated by Lin and Wang. The CAP is intended to identify creativity levels among students in grades 4 to 12 [41]. The two subtests of the CAP-the Test of Divergent Thinking (TDT) and the Test of Divergent Feeling (TDF) - were investigated for relationships with the Divergent Thinking and Motivation subscales measured by the CPSAI, respectively. In the TDT, examinees completed drawing 12 different figures, based on the lines presupplied in each item, and gave titles to their drawings. Their final products were scored based on fluency, flexibility, originality, collaboration, openness, and title. The TDF is a 3-point Likert scale questionnaire that asks students' preference on statements in four subscales: curiosity; risk taking; desire for complexity; and imagination.

In this study, 31 students' responses were randomly selected and rated by an external rater to test the interrater reliability of the TDT, which was found to reach .91. Additionally, the alpha value for internal-consistency was .85 for the TDF and .64 for the TDT. The overall score on the TDT significantly correlated with the subscale scores for fluency $(r=.49, p=0.01)$, originality $(r=.58, p=0.01)$, openness $(r=.84, p=0.01)$, elaboration $(r=.82, p=0.01)$, and titles $(r=.60, p=0.01)$, but not with flexibility.

The Critical Thinking Test- (CTT-) I. The CTT-I measures critical thinking skills of 5th- to 12th-grade students, with 25 multiple-choice questions that represent five critical thinking skills (Identification of Assumptions; Induction; Deduction; Interpretation; and Evaluation of Arguments) [39]. The test has previously been validated with 2,228 elementary students, and the alpha, using the ITEMAN item analysis, was .76. Cronbach's alpha for each subscale ranged from .32 to .49. Correlations between the overall score and the subscale scores ranged from .23 to .45 ( $p<0.001)$. In this study, Cronbach's $\alpha$ of the overall test was .57. The overall score of the CTT-I significantly correlated with Identification of Assumptions $(r=.61, p<0.001)$; Induction $(r=.61$, $p<0.001)$; Deduction $(r=.67, p<0.001)$; Interpretation $(r=.60, p<0.001)$; and Evaluation of Arguments $(r=.45$, $p<0.001)$.

The Inventory of Parental Influence (IPI). The IPI is a crosscultural, self-reported instrument designed to identify students' perceptions of family processes, including parental pressure; support; help; pressure toward intellectual development; monitoring; and communication [40]. A positive family process has been found to significantly influence students' creative problem-solving ability [42]. In addition, support and pressure toward intellectual development have been observed to significantly affect former Science Olympians' academic achievements [42]. Cho and Campbell [42] validated the instrument with 757 past Science Olympians and obtained internal-consistency reliability for the scales of support $(r=.63)$ and of pressure toward intellectual development $(r=.80)$.

In this study, the relationships between the Environment subscale of the CPSAI and the subscales of Pressure toward Intellectual Development and of Support were investigated. This is because a positive family process, which consists of support and pressure toward intellectual development, is significantly correlated with creative problem-solving in math [43]. In this study, the internal-consistencies of the Support and Pressure toward Intellectual Development subscales were $\alpha=.75$ and $\alpha=.76$, respectively.

In summary, the TDT and TDF subtests of the CAP, the CTT-I, and the IPI were administered to determine whether the attributes measured by the CPSAI subscales are similar to those measured by these established instruments. Scores on general and domain-specific knowledge and skills measured by the CPSAI were compared with students' GPAs and academic achievements, respectively.

2.4. Data Collection. The MCPSAT, CPSAI, CAP, CTT-I, and IPI were administered during two one-hour sessions over two weeks, with the MCPSAT and CPSAI in the first week and the CAP, CTT-I, and IPI in the second week. All instruments were administered by the author, with assistance from teachers at the schools. Students' math achievement test scores, which are administered three times per semester, and GPAs from the previous semester were used as measures of domain-specific and general knowledge and skills.

2.5. Data Analysis. A confirmatory factor analysis was performed to determine if the relationships between the variables in Cho's model resemble the relationships between the variables in the observed data. In addition, internalconsistency between the overall scores and the score for each subscale of the CPSAI items and correlation coefficients among the subscale scores were analyzed. The correlation coefficients between the sub-scores and total score of the CPSAI were computed. Additionally, the scores from the CAP, CTT-I, and IPI were analyzed in comparison with the respective subscales of the CPSAI. A cluster analysis based on participants' responses on the CPSAI was performed to determine the typical typology of creative problem-solving ability in Taiwanese students. An analysis of variance (ANOVA) and follow-up post hoc tests were carried out to investigate the mean differences in the five attributes (divergent thinking; convergent thinking; motivation; general knowledge; and environment) and to investigate differences in the scores on the MCPSAT between the clusters.

\section{Results}

3.1. Structural Validity and Reliability. Confirmatory factor analysis was conducted to examine if the hypothesized fivefactor structure, which was believed to underlie the factors in the CPSAI, fits the data. Hypothesized models were assessed 
TABLE 2: Mean, SD, and $n$ among the students' responses on five subscales and CPSAI total score.

\begin{tabular}{lccc}
\hline Variables & $N$ & $M$ & SD \\
\hline Divergent Thinking & 359 & 28.30 & 8.34 \\
Convergent Thinking & 342 & 22.17 & 6.63 \\
Motivation & 338 & 19.26 & 5.58 \\
General Knowledge & 335 & 14.65 & 5.22 \\
Environment & 351 & 36.40 & 10.94 \\
\hline
\end{tabular}

by AMOS maximum likelihood factor analysis and evaluated for four measures of fit: (a) chi-square test; (b) Comparative Fit Index (CFI); (c) Normed Fit Index (NFI); and (d) Root Mean Square Error of Approximation (RMSEA).

The Divergent Thinking scale showed a good fit $\left(\chi^{2}=\right.$ $67.89(35,281), p<0.001$, CFI $=.960$, NFI $=.923$, and RMSEA = .058). The Convergent Thinking scale moderately fit the observed data $\left(\chi^{2}=77.08(20,281), p<0.001\right.$, CFI $=.935$, NFI $=.914$, and RMSEA $=.101)$. The model for the Motivation scale was a reasonably good fit $\left(\chi^{2}=15.214(9\right.$, 281), $p=0.085$, CFI $=.983$, NFI $=.960$, and RMSEA $=$ .050). There was a good fit for the hypothesized model for General Knowledge and Skills $\left(\chi^{2}=4.647(5, N=281)\right.$, $p=0.460, \mathrm{CFI}=1.00, \mathrm{NFI}=.992$, and RMSEA $=.000)$. For the Environment scale, the chi-square yielded an undesirable value $\left(\chi^{2}=153.121(44, N=281), p<0.001\right)$, but a moderate fit with the observed data was seen $(\mathrm{CFI}=.924$, NFI $=.897$, and RMSEA $=.094)$. Overall, the findings of the confirmatory factor analysis yielded a good to moderate fit between the five-factor structure model and the observed data.

Correlation coefficients were computed for the five subscales. Tables 2 and 3 present the means and standard deviations, as well as the correlational analysis in which statistically significant correlations ranged from .45 to .70 $(p<0.01)$. The correlation between the Divergent Thinking and Convergent Thinking scales was the highest $(r=.70, p<$ $0.01)$. The Motivation scale correlated higher with Divergent Thinking $(r=.64, p<0.01)$ and Convergent Thinking $(r=$ .64, $p<0.01)$ compared to General Knowledge and Skills $(r=.50, p<0.01)$ and Environment $(r=.55, p<0.01)$. In terms of the relationship between the overall CPSAI score and each subscale, Divergent Thinking $(r=.84, p<0.001)$ and Convergent Thinking $(r=.84, p<0.001)$ correlated higher with the CPSAI total score, while Environment $(r=.80$, $p<0.001)$, Motivation $(r=.78, p<0.001)$, and General Knowledge and Skills $(r=.75, p<0.001)$ appeared to be slightly less correlated.

To assess whether the items for each scale yielded reliable data, Cronbach alphas were computed. The alpha values for Divergent Thinking $(\alpha=.87)$, Convergent Thinking $(\alpha=$ $.84)$, Environment ( $\alpha=.89)$, Motivation $(\alpha=.79)$, and General Knowledge and Skills $(\alpha=.85)$ indicated that their respective items had good internal-consistency. Furthermore, the internal-consistency of the items overall was good $(\alpha=$ $.85)$.
3.2. Relationship between CPS Attributes and the Established Instruments. The Pearson correlation coefficients between Divergent Thinking and TDT $(r=.15, p<0.01)$, Convergent Thinking and the CTT-I $(r=.19, p<0.01)$, Motivation and the TDF $(r=.40, p<0.01)$, Environment and positive IPI I $(r=.61, p<0.01)$, and General Knowledge and Skills and academic achievement $(r=.40, p=0.01)$ indicated that the scores on the established instruments and academic achievement were related to the scores on the attributes of CPSAI. Medium to large effect sizes were found in the associations. The correlation coefficient between the Divergent Thinking scale of CPSAI and the TDT was the lowest. The content of the Divergent Thinking scale of the CPSAI measures math problem-solving situations, whereas the TDT measures divergent thinking in drawing figures.

3.3. Differences in Attributes among Different Math Creativity Groups. A hierarchical cluster analysis was performed to identify relatively homogenous groups, based on students' responses in the scales of Divergent thinking, Convergent thinking, Motivation, General Knowledge and Skills, and Environment. After excluding missing values, $73 \%$ of the samples were included in the cluster analysis. Three clusters were found to be the most appropriate, as they yielded the most statistically significant differences among the clusters. These three clusters were eventually (see below) labeled Low, Medium, and High.

A one-way ANOVA was conducted to examine the differences in the five attributes among the three clusters. Levene tests on the first one-way ANOVA were significant for the subscales of Divergent Thinking and Motivation, which indicated violations of the assumption of homogeneity of variances. Therefore, Games-Howell tests were performed for these two subscales, whereas Tukey HSD tests were conducted for the subscales of Convergent Thinking, Environment, and General Knowledge and Skills.

Table 4 shows statistically significant differences between the three clusters in Convergent Thinking $(F(2,296)=166.93$, $p<0.001)$, Environment $(F(2,296)=173.68, p<$ $0.001)$, and General Knowledge and Skills $(F(2,296)=99.13$, $p<0.001)$. The means for Convergent Thinking, Environment, General Knowledge and Skills, Divergent Thinking, and Motivation in Cluster 3 were the highest among the three clusters, whereas Cluster 1 showed the lowest means across all five attributes. Post hoc Tukey tests indicated that the mean differences between pairs of clusters were significant in each of these five subscales $(p<0.05)$. Clusters 1, 2, and 3 were labeled as Low Attribute (LA), Medium Attribute (MA), and High Attribute (HA) based on their mean scores in all five subscales (see Figure 1). The High Attribute (HA) group had at least above medium levels in Divergent Thinking, Convergent Thinking, Environment, and General Knowledge. In addition, the HA students tended to have very high motivation when solving new and difficult problems. On the other hand, mean scores of the Low Attribute (LA) group were the lowest in each subscale among the three clusters. Furthermore, the HA students showed higher scores in Motivation than did the MA and LA students. 
TABLE 3: Correlation coefficients between students' responses on five subscales and CPSAI overall score.

\begin{tabular}{|c|c|c|c|c|c|c|}
\hline Variables & $\begin{array}{l}\text { Divergent } \\
\text { Thinking }\end{array}$ & $\begin{array}{c}\text { Convergent } \\
\text { Thinking }\end{array}$ & Motivation & $\begin{array}{c}\text { General } \\
\text { Knowledge and } \\
\text { Skills }\end{array}$ & Environment & $\begin{array}{l}\text { CPSAI total } \\
\text { score }\end{array}$ \\
\hline Divergent Thinking & - & $.70^{* *}$ & $.64^{* *}$ & $.56^{* *}$ & $.48^{* *}$ & $.84^{* *}$ \\
\hline Convergent Thinking & & - & $.63^{* *}$ & $.57^{* *}$ & $.53^{* *}$ & $.84^{* *}$ \\
\hline Motivation & & & - & $.55^{* *}$ & $.50^{* *}$ & $.78^{* *}$ \\
\hline General Knowledge & & & & - & $.45^{* *}$ & $.75^{* *}$ \\
\hline Environment & & & & & - & $.80^{* *}$ \\
\hline CPSAI total score & & & & & & - \\
\hline
\end{tabular}

TABLE 4: One-way analysis of variance results for group differences in Convergent Thinking, Environment, General Knowledge and Skills, Divergent Thinking, and Motivation among the three attribute pattern clusters.

\begin{tabular}{|c|c|c|c|c|c|c|c|}
\hline & Cluster & $N$ & $M$ & $\mathrm{SD}$ & $F$ & $p$ & Tukey \\
\hline \multirow{4}{*}{$\begin{array}{l}\text { Convergent Thinking } \\
\text { (8 items) }\end{array}$} & Cluster 1 & 62 & 1.93 & 5.65 & \multirow{4}{*}{166.93} & \multirow{4}{*}{0.000} & \multirow{4}{*}{$\begin{array}{l}\text { Cluster } 2>\text { Cluster } 1^{*} \\
\text { Cluster } 3>\text { Cluster } 1^{*} \\
\text { Cluster } 3>\text { Cluster } 2^{*}\end{array}$} \\
\hline & Cluster 2 & 113 & 2.54 & 4.37 & & & \\
\hline & Cluster 3 & 124 & 3.47 & 4.17 & & & \\
\hline & Total & 299 & 2.80 & 6.67 & & & \\
\hline \multirow{4}{*}{$\begin{array}{l}\text { Environment } \\
\text { (11 items) }\end{array}$} & Cluster 1 & 62 & 1.93 & 6.70 & \multirow{4}{*}{173.68} & \multirow{4}{*}{0.000} & \multirow{4}{*}{$\begin{array}{l}\text { Cluster } 2>\text { Cluster } 1^{*} \\
\text { Cluster } 3>\text { Cluster } 1^{*} \\
\text { Cluster } 3>\text { Cluster } 2^{*}\end{array}$} \\
\hline & Cluster 2 & 113 & 3.38 & 7.58 & & & \\
\hline & Cluster 3 & 124 & 3.93 & 8.05 & & & \\
\hline & Total & 299 & 3.31 & 11.18 & & & \\
\hline \multirow{5}{*}{$\begin{array}{l}\text { General Knowledge } \\
\text { and Skills } \\
\text { (5 items) }\end{array}$} & Cluster 1 & 62 & 1.93 & 3.81 & \multirow{4}{*}{99.13} & \multirow{4}{*}{0.000} & \multirow{4}{*}{$\begin{array}{l}\text { Cluster } 2>\text { Cluster } 1^{*} \\
\text { Cluster } 3>\text { Cluster } 1 \\
\text { Cluster } 3>\text { Cluster } 2\end{array}$} \\
\hline & Cluster 2 & 113 & 2.70 & 4.19 & & & \\
\hline & Cluster 3 & 124 & 3.60 & 3.81 & & & \\
\hline & Total & 299 & 2.92 & 5.10 & & & \\
\hline & Cluster & $N$ & $M$ & $\mathrm{SD}$ & $F$ & $p$ & Games-Howell \\
\hline \multirow{4}{*}{$\begin{array}{l}\text { Divergent Thinking } \\
\text { (10 items) }\end{array}$} & Cluster 1 & 62 & 2.07 & 6.18 & \multirow{4}{*}{267.43} & \multirow{4}{*}{0.000} & \multirow{4}{*}{$\begin{array}{l}\text { Cluster } 2>\text { Cluster } 1^{*} \\
\text { Cluster } 3>\text { Cluster } 1^{*} \\
\text { Cluster } 3>\text { Cluster } 2^{*}\end{array}$} \\
\hline & Cluster 2 & 113 & 2.37 & 4.08 & & & \\
\hline & Cluster 3 & 124 & 3.62 & 5.23 & & & \\
\hline & Total & 299 & 2.93 & 8.44 & & & \\
\hline \multirow{4}{*}{$\begin{array}{l}\text { Motivation } \\
\text { (7 items) }\end{array}$} & Cluster 1 & 62 & 2.10 & 5.23 & \multirow{4}{*}{103.93} & \multirow{4}{*}{0.000} & \multirow{4}{*}{$\begin{array}{l}\text { Cluster } 2>\text { Cluster } 1^{*} \\
\text { Cluster } 3>\text { Cluster } 1^{*} \\
\text { Cluster } 3>\text { Cluster } 2^{*}\end{array}$} \\
\hline & Cluster 2 & 113 & 2.52 & 3.68 & & & \\
\hline & Cluster 3 & 124 & 3.34 & 4.09 & & & \\
\hline & Total & 299 & & 5.48 & & & \\
\hline
\end{tabular}

Note. $^{*} p<0.05$.

Students in the Medium Attribute (MA) group had higher levels of each attribute than those in the LA group but had lower levels in Divergent Thinking, ConvergentThinking, Motivation, Environment, and Knowledge than did those in the HA group.

Significant differences were found among the three clusters in their MCPSAT scores, $F(2,290)=9.69, p=0.00$ (see Table 5). The HA students scored highest on the MCPSAT and the LA students scored the lowest. Post hoc Tukey tests indicated that the HA group significantly differed from the MA and LA students, while differences between the LA and MA groups were insignificant.

\section{Discussion}

This study aimed to investigate typology of different creative problem-solving groups. A newly devised instrument, the CPSAI, with five different subscales (Divergent Thinking; 
TABLE 5: Mean and SD of MCPSAT and group differences between three clusters.

\begin{tabular}{cccccccr}
\hline & Clusters & $n$ & $M$ & SD & $F$ & $p$ & Tukey \\
\hline \multirow{3}{*}{ MCPSAT } & LA & 60 & 27.52 & 21.50 & & & \\
& MA & 110 & 28.61 & 17.01 & & \\
& HA & 123 & 38 & 18.98 & & 0.000 & HA $^{\prime}$ MA $^{*}$ \\
& Total & 293 & 29.26 & 32.33 & & & \\
\hline
\end{tabular}

Note. $^{*} p<0.05$.

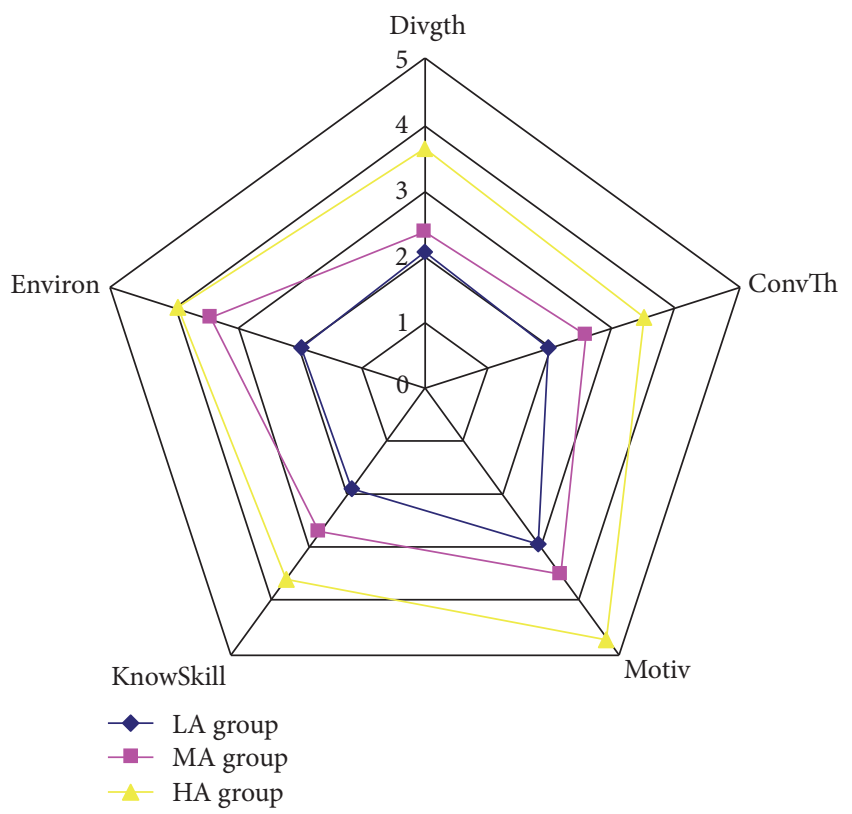

FIGURE 1: Creative problem-solving attribute combination patterns for HA, MA, and LA clusters.

Convergent Thinking; Motivation; General Knowledge; and Environment), was utilized in this study. Several analyses were computed to investigate the reliability and validity of the CPSAI, as well as its correlation with established instruments such as the MCPSAT, CAP, CTT-I, and IPI. Those instruments measure creative problem-solving in math, divergent thinking, convergent thinking, and family processes, respectively.

According to the results of the confirmatory factor analysis, most of the subscales yielded a reasonable fit, save for the Convergent Thinking scale. Further clarification may be needed for items measuring convergent thinking, such as rephrasing them in a way that allows examinees to understand them more readily. In addition, there have been studies indicating the importance of domain-specific knowledge in creativity $[20,23]$. Therefore, the concept of domain specificity may need to be incorporated into the test questions for convergent thinking, motivation, and divergent thinking in the CPSAI in the future. As Brophy [34] pointed out, individuals constantly alternate between ideation and evaluation during the creative problem-solving process, such that these two types of cognitive thinking may work in a "zigzag pattern." This may have caused difficulty for the participating fifth and sixth graders in distinguishing between the divergent and convergent thinking processes. Divergent thinking and convergent thinking may not be processes that are exclusive of each other. While an individual explores alternatives, convergent thinking is also required to evaluate previous solutions or ideas and, based on the evaluation results, they will continue to or stop searching for new alternatives [34]. Future research may need to be conducted, with participants with higher levels of cognitive maturity.

Values for internal-consistency on each subscale ranged from .79 to .89 , indicating good reliability. Further, the items interrelated well enough to be summed as a composite variable, and each of the five factors was measured well by its subscale items. The subscales statistically correlated with each other, ranging from .45 to .70, suggesting moderate to high relationships between them. The structures of the subscales shared some common ground yet were unique from each other, confirming Cropley's claim that these two types of cognitive processes (convergent and divergent thinking) are both used to generate ideas, but in different fashions [29]. Divergent Thinking and Convergent Thinking were the most highly correlated among the subscales. This corresponds to the results of the factor analysis, which revealed that these two concepts were not easily distinguished by the participants.

The results of the correlation analyses between the scores on the subscales and established instruments ranged between .20 and .60, signifying medium to large relationships, suggesting that there are similarities as well as differences between the subscales of the CPSAI and the other established instruments. For example, the Divergent Thinking scale had a significant but low correlation with TDT. This result can be explained by the domain specificity of creativity and divergent thinking. Baer theorized that creativity is domainspecific, such that creativity in one field cannot be transferred to another field, based on the low correlations among products in five tasks (telling stories; writing stories; writing poems; writing mathematical word problems; and making collages) [44-46]. In other words, an individual's creativity in drawing figures as tested in the TDT may not transfer to creativity in solving math problems.

The cluster analysis yielded three patterns of attributes, with the HA group having the highest mean scores across all of the attributes, followed by the MA group, and then the LA group. In addition, participants in the HA group 
acquired the highest scores on the MCPSAT, followed by students in the MA and LA groups, respectively. Although the three groups significantly differed from each other across attributes, only the MCPSAT score of the HA group was significantly different from the other two groups; the MA and LA groups had similar scores on the MCPSAT. This implies that there might be a threshold effect required for attributes in creative problem-solving to have an impact. In other words, unless students have at least a threshold level (the midpoint of the 5-point scale) in all of the attributes, differences in these attributes below the threshold level do not result in a difference in actual creative problem-solving.

This empirical finding of the present study supports Cho's Dynamic System Model of Creative Problem-Solving Ability $[23,24,30]$, in which all attributes of creative problem-solving are equally important and have to be adequately developed before superior creativity can manifest. More importantly, these results imply the importance of balanced development among attributes. No particular attribute stands out from the rest. Instead, attributes of creative problem-solving need to be developed equally to certain levels, as supported by Cho, who claimed that not only do attributes of creative problem-solving interact with each other to help solve problems in a creative manner during the process, but also creative problem-solving is an organic system that cannot develop properly without the balanced development of these attributes [24]. Therefore, two principles in the nurturing and development of creative problem-solving are as follows: (a) all attributes are equally important and (b) these attributes must all be developed to a certain level in order for an individual to perform creative problem-solving [47]. Moreover, past studies have discussed the threshold theory with regard to intelligence and creativity [48-50], but not the threshold theory's effect on other specific attributes. Therefore, these current findings should be further investigated to determine their generalizability.

\section{Conclusion}

In this evolving environment, creative problem-solving is essential for human beings to solve the complex challenges we face [3]. It is critical to equip our children with creative problem-solving abilities. This study investigated the typology of math creative problem-solving ability. Most importantly, the results of this study may have shown a threshold effect for creative problem-solving attributes, meaning that one has to achieve a certain level in divergent thinking, convergent thinking, motivation, environment, and knowledge and skills in order for them to have an impact on creative problem-solving ability. This study showed that the attributes in Cho's $[23,24,30]$ model are equally important and need to be developed equally to certain levels, thus contributing to the existing knowledge regarding creative problem-solving.

\section{Conflicts of Interest}

The author declares that there are no conflicts of interest regarding publishing this paper.

\section{References}

[1] E. C. Selby, E. J. Shaw, and J. C. Houts, "The creative personality," Gifted Child Quarterly, vol. 49, no. 4, p. 300, 2005.

[2] D. K. Simonton, "Creativity: Cognitive, personal, developmental, and social aspects," American Psychologist, vol. 55, no. 1, pp. 151-158, 2000.

[3] World Economic Forum (2015), "New Vision for Education: Unlocking the Potential of Technology," http://www3.weforum .org/docs/WEFUSA_NewVisionforEducation_Report2015.pdf.

[4] D. J. Treffinger, E. C. Selby, and S. G. Isaksen, "Understanding individual problem-solving style: A key to learning and applying creative problem solving," Learning and Individual Differences, vol. 18, no. 4, pp. 390-401, 2008.

[5] J. Davidson and R. Sternberg, "Smart problem solving; How metacognition helps," in Metacognition in Educational Theory, D. J. Hacker, J. Dunlosky, and A. C. Graesser, Eds., pp. 47-68, Erlbaum, Mahwah, NJ, 1998.

[6] H. Kim, S. Cho, and D. Ahn, "Development of mathematical creative problem solving ability test for identification of the gifted in math," Gifted Educational International, vol. 18, no. 2, pp. 164-175, 2003.

[7] G. Kaufmann, "Problem solving and creativity," in Innovation: A cross-disciplinary perspective, K. Gronhaug, Ed., pp. 87-137, Norwegian University Press, Oslo, Norway, 1988.

[8] J. C. Kaufman and R. J. Sternberg, "Resource review: Creativity," Change, July/August, pp. 55-58, 2007.

[9] A. M. Runscio and T. M. Amabile, "Effects of instructional styles on problem-solving creativity," Creativity Research Journal, vol. 12, no. 4, pp. 251-266, 1999.

[10] M. Csikzentmihalyi and J. W. Getzels, "Discovery-oriented behavior and the originality of creative products: A study with artists," Journal of Personality and Socical Psychology, vol. 19, pp. 47-52, 1971.

[11] J. P. Guilford, Creativity and its cultivation, Harper, New York, 1959.

[12] J. C. Houtz, "Creative problem solving in the classroom: Contributions of four psychological approaches," in Problem finding, problem solving, and creativity, M. Runco, Ed., pp. 4076, Ablex Publishing Corporation, Norwood, New Jersey, 1994.

[13] E. P. Torrance, Guiding creative talent, Prentice-Hall, Englewood Cliffs, NJ, 1962.

[14] D. J. Treffinger, "Myth 5: Creativity is too difficult to measure," Gifted Child Quarterly, vol. 53, no. 4, pp. 245-247, 2009.

[15] M. A. Runco, G. Dow, and W. R. Smith, "Information, experience, and divergent thinking: An empirical test," Creativity Research Journal, vol. 18, no. 3, pp. 269-277, 2006.

[16] X. Ai, "Creativity and academic achievement: An investigation of gender differences," Creativity Research Journal, vol. 12, no. 4, pp. 329-337, 1999.

[17] M. Csikszentmihalyi, Creativity, Flow and the psychology of discovery and invention, Harper Perennial, New York, 1996.

[18] R. J. Sternberg and T. I. Lubart, Defying the crowd: Cultivating creativity in the culture of conformity, Free press, New York, 1995.

[19] H. Gardner, Creating minds: An anatomy of creativity, Basic, New York, 1993.

[20] T. M. Amabile, The Social Psychology of Creativity, Springer New York, New York, NY, USA, 1983a.

[21] J. C. Kaufman, C. A. Gentile, and J. Baer, "Do gifted student writers and creative writing experts rate creativity the same way?” Gifted Child Quarterly, vol. 49, no. 3, pp. 260-265, 2005. 
[22] J. P. Guilford, Convergent-production Abilities: The nature of human intelligence, McGraw-Hill, New York, USA, 1967.

[23] T. M. Amabile, "The social psychology of creativity: A componential conceptualization," Journal of Personality and Social Psychology, vol. 45, no. 2, pp. 357-376, 1983.

[24] S. Cho, "Creative problem solving in science: Divergent, convergent, or both?" in Proceedings of the 7th Asia-pacific Conference on Giftedness, U. Anuruthwong and C. Piboonchol, Eds., pp. 169-174, October Printing, Bangkok, Thailand, 2003.

[25] S. Cho, "Nurturing creative problem solving ability of the gifted in Confucian society," The Journal of Gifted/Talented Education, vol. 17, no. 2, pp. 392-411, 2007.

[26] J. P. Guilford, "Creativity," American Psychologist, vol. 5, no. 9, pp. 444-454, 1950.

[27] R. J. Sternberg, "A three-facet model of creativity", in The nature of creativity: Contemporary psychological perspectives, J. R. Sternberg, Ed., pp. 125-147, Cambridge University Press, New York, 1988.

[28] E. P. Torrance, "The nature of creativity as manifest in its testing," in The nature of creativity: Contemporary psychological perspectives, J. R. Sternberg, Ed., pp. 43-75, Cambridge University Press, New York, USA, 1988.

[29] K. Urban, "Toward a componential model of creativity," in Creative intelligence: Toward theoretic integration, D. Ambrose, L. M. Cohen, and A. J. Tannenbaum, Eds., pp. 81-112, Hampton Press, Cresskill, NJ, 2003.

[30] S. Cho, "Education of creativity," in in proceedings of Keynote speech at the 8th International Conference on Thinking, Edmonton, Canada, July 1999.

[31] J. S. Renzulli, “The three-ring conception of giftedness: A theory and identification plan for promoting creativity productivity," Connecticut: The National Research Center on the Gifted and Talented, 2008.

[32] J. F. Feldhusen, "Creativity: The knowledge base and children," High Ability Studies, vol. 13, no. 2, pp. 179-183, 2002.

[33] A. Cropley, "In praise of convergent thinking," Creativity Research Journal, vol. 18, no. 3, pp. 391-404, 2006.

[34] D. R. Brophy, "Understanding, measuring, and enhancing individual creative problem-solving efforts," Creativity Research Journal, vol. 11, no. 2, pp. 123-150, 1998.

[35] R. L. Firestien and D. J. Treffinger, "Creative problem solving: Guidelines and resources for effective facilitation," $G / C / T /$ magazine, pp. 35-39, 1989.

[36] S. G. Isaksen, "CPS:, Linking creativity and problem solving," in Problem solving and cognitive process: A festschrift in honour of Kjell Raaheim, T. Kaufmann, T. Helstrup, and K. H. Teigen, Eds., pp. 145-181, Fagbokforlagent Vigmostad \& Bjorke AS, Norway, 1995.

[37] D. J. Treffinger, "Creative Problem Solving: Overview and educational implications," Educational Psychology Review, vol. 7, no. 3, pp. 301-312, 1995.

[38] F. E. Williams, Creativity assessment packet, PRO-ED, Inc, Austin, Texas, 1980.

[39] Y. Yen, The Critical Thinking Test-Level I, The Psychological Publishing Co., Ltd, Taipei, Taiwan, 2003.

[40] J. R. Campbell, "Developing cross-cultural/cross-national instruments: Using cross-national methods and procedures," International Journal of Educational Research, vol. 21, no. 7, pp. 675-684, 1994.
[41] S. Lin and M. Wang, Creativity assessment packet (Taiwan Edition), The Psychological Publishing Co., Ltd, 1994, http:// www.psy.com.tw/product_desc.php?cPath=26\&amp;cate_id= 45\&amp;products_id $=118$.

[42] S. Cho and J. R. Campbell, "Cross sectional analyses on the relationship between family processes and academic achievements of the gifted and Olympians," Roeper Review, 2011.

[43] S. Cho and C. Lin, "Influences of family process and affective characteristics on preference for creative problem solving of the gifted and Olympians," Roeper Review, 2011.

[44] J. Baer, Divergent thinking and creativity: A task-specific approach, Lawrence Erlbaum Associates, Inc, Hillsdale, NJ, 1993.

[45] J. Baer, "Divergent Thinking is not a General Trait: A Multidomain Training Experiment," Creativity Research Journal, vol. 7, no. 1, pp. 35-46, 1994.

[46] J. Baer, "The effects of task-specific divergent-thinking training," Journal of Creative Behavior, vol. 30, no. 3, pp. 183-187, 1996.

[47] C. Y. Lin and Cho, "Threshold effects of creative problem solving attributes on creative performance in math of Taiwanese upper elementary school students," in Proceedings of the Threshold effects of creative problem solving attributes on creative performance in math of Taiwanese upper elementary school students, Vancouver, Canada, 2012.

[48] F. Preckel, H. Holling, and M. Wiese, "Relationship of intelligence and creativity in gifted and non-gifted students: An investigation of threshold theory," Personality and Individual Differences, vol. 40, no. 1, pp. 159-170, 2006.

[49] K. H. Kim, "Can Only Intelligent People Be Creative? A MetaAnalysis," Journal of Secondary Gifted Education, vol. 16, no. 2-3, pp. 57-66, 2005.

[50] E. Jauk, M. Benedek, B. Dunst, and A. C. Neubauer, "The relationship between intelligence and creativity: New support for the threshold hypothesis by means of empirical breakpoint detection," Intelligence, vol. 41, no. 4, pp. 212-221, 2013. 


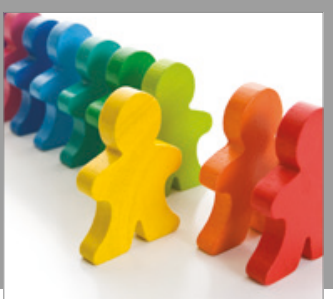

Autism

Research and Treatment
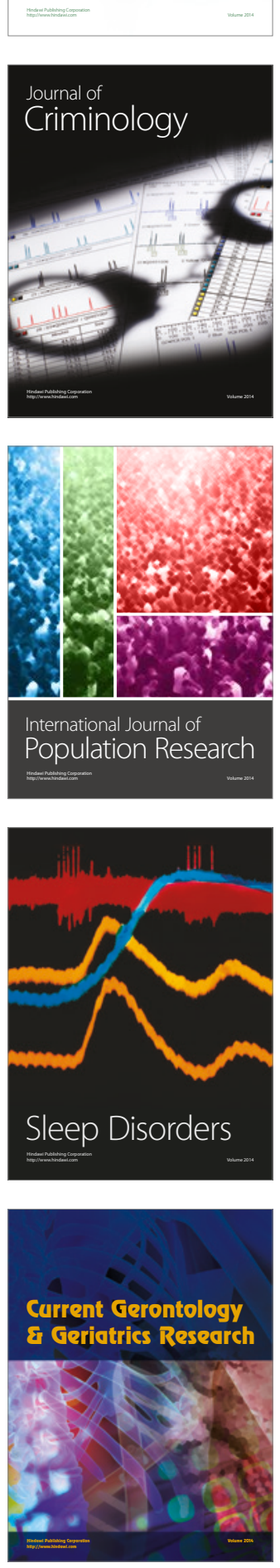

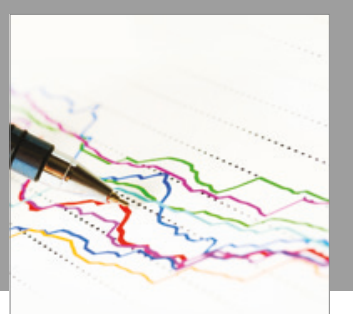

Economics

Research International

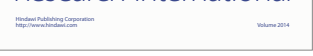

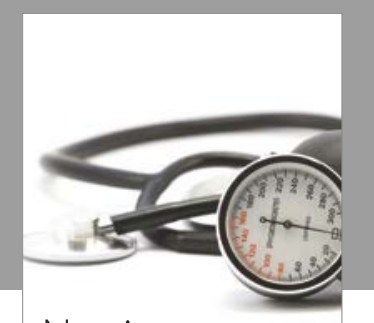

Nursing

Research and Practice

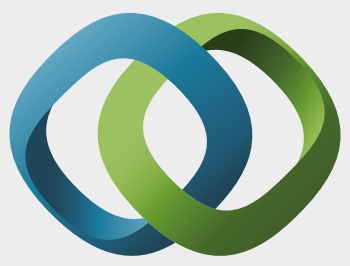

\section{Hindawi}

Submit your manuscripts at

https://www.hindawi.com
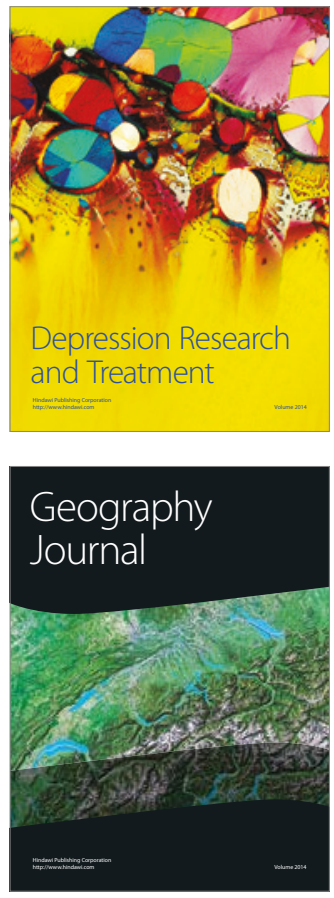
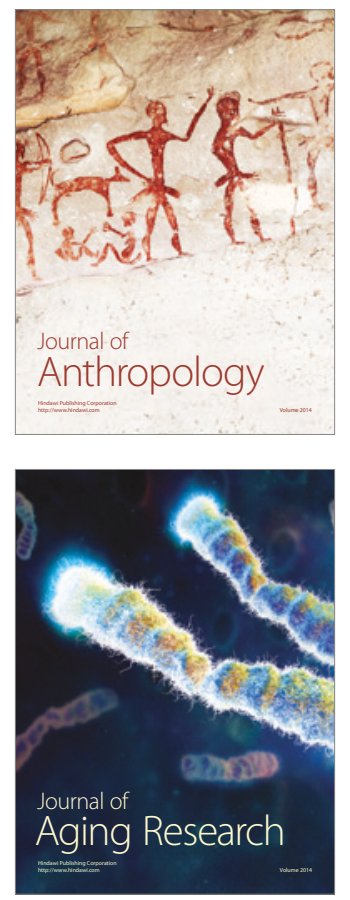
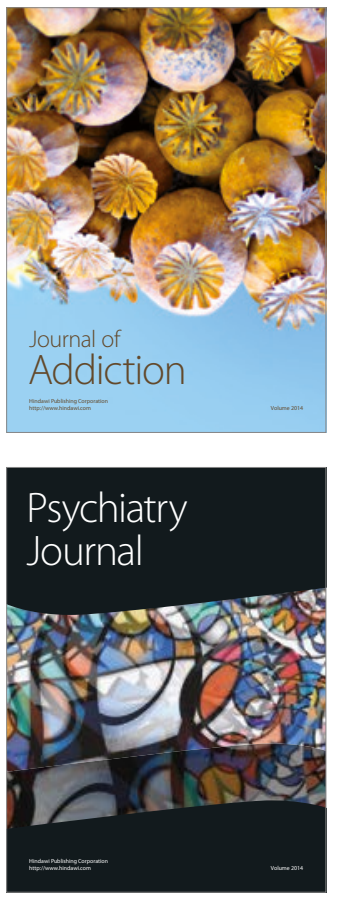

Child Development

Research

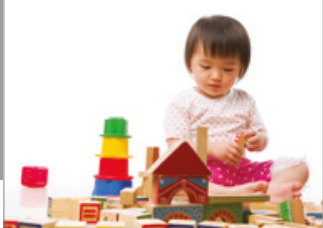

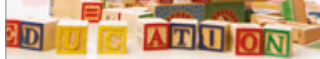
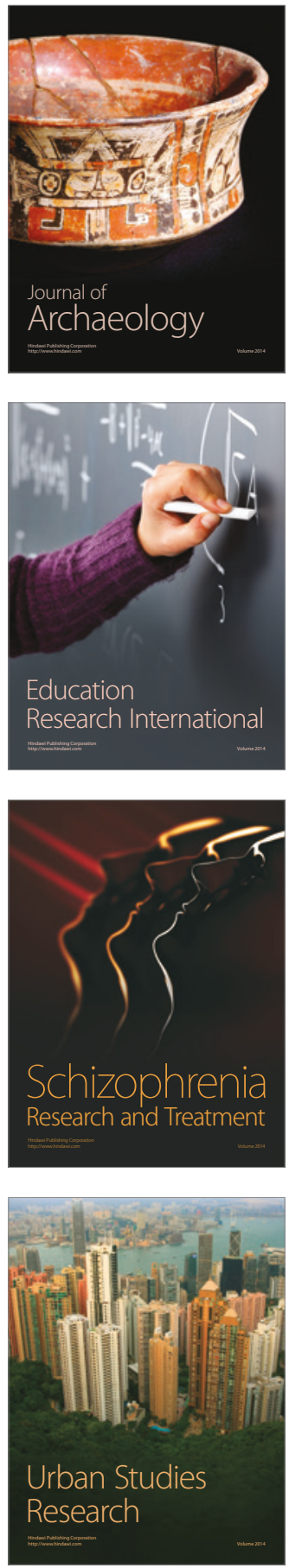